\title{
Oral Hairy Leukoplakia Associated With the Use of Adalimumab
}

\author{
Molly B. Kitley, MD; Timothy Devitt, DMD; Don-John Summerlin, DMD; Carrie L. Davis, MD
}

\section{PRACTICE POINTS}

- Workup for new-onset oral hairy leukoplakia should include a comprehensive medication history.

- Oral hairy leukoplakia is an uncommon side effect of adalimumab.

To the Editor:

Oral hairy leukoplakia (OHL) is an Epstein-Barr virus (EBV)-mediated mucocutaneous disease that often involves the lingual epithelium. The lateral portions of the tongue are the most commonly affected sites. The lesions often are described as asymptomatic, white, corrugated patches or plaques that are unable to be scraped off. ${ }^{1}$ Oral hairy leukoplakia was first identified in 1984 and was considered to be associated with AIDS. ${ }^{2}$ An association between the presence of $\mathrm{OHL}$ and the degree of immunosuppression as well as the severity of human immunodeficiency virus (HIV) has been reported. ${ }^{3}$ Although OHL initially was considered to be pathognomonic for HIV, it has since been described in multiple other immunosuppressive conditions. ${ }^{4}$ Numerous medical conditions and combinations of immunosuppressive medications have been associated with OHL in patients who were HIV negative. ${ }^{5}$

Adalimumab is an injectable human IgG1 recombinant antibody to tumor necrosis factor $\alpha$ (TNF- $\alpha){ }^{6}$ It currently is approved by the US Food and Drug
Administration for the treatment of rheumatoid arthritis, juvenile idiopathic arthritis, psoriatic arthritis, ankylosing spondylitis, adult and pediatric Crohn disease, ulcerative colitis, noninfectious uveitis, hidradenitis suppurativa, and plaque psoriasis. ${ }^{7}$ We report a case of OHL associated with the use of adalimumab.

A 47-year-old woman initially presented with chronic plaque-type psoriasis. Her medical history was notable for bipolar disorder, migraines, hypertension, and tobacco use. The patient's psoriasis initially was well controlled on a regimen of topical steroids and methotrexate; however, methotrexate was stopped after 2.5 years due to a mildly elevated alanine aminotransferase level, as well as an abnormal liver biopsy showing mildly active (grade 1 of 3) steatohepatitis with portal chronic inflammation, pericellular fibrosis, and portal and focal periportal fibrosis (stage 1-2 of 4). The patient and her dermatologist were uncomfortable continuing methotrexate with these findings. After baseline screening including a negative purified protein derivative skin test, adalimumab was initiated. A loading dose of $80 \mathrm{mg}$ subcutaneously (SQ) was given, followed by adalimumab $40 \mathrm{mg}$ SQ 1 week later and $40 \mathrm{mg}$ every other week as maintenance.

The patient's psoriasis was well controlled with adalimumab for 22 months, but she then developed a thin white plaque on the right lateral tongue (Figure 1). An incisional biopsy of the tongue performed by an oral surgeon revealed hyperkeratosis with Candida colonization and viral cytopathic effect (Figure 2). An EBV DNA in situ

Dr. Kitley is from the Department of Dermatology, University of Minnesota, Minneapolis. Dr. Summerlin is from the Department of Pathology \& Laboratory Medicine, Indiana University School of Medicine, Indianapolis. Dr. Devitt is from Bloomington Oral Surgery, Indiana. Dr. Davis is from the Dermatology Center of Southern Indiana, Bloomington, and the Indiana University School of Medicine, Bloomington.

The authors report no conflict of interest.

Correspondence: Carrie L. Davis, MD, 1200 S Rogers St, Bloomington, IN 47401 (carriedavismd@gmail.com).

doi:10.12788/cutis.0119 


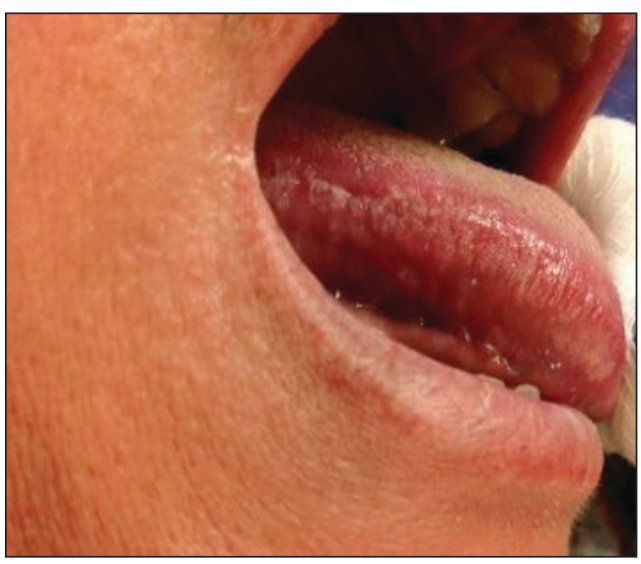

FIGURE 1. Oral hairy leukoplakia. A corrugated white plaque on the right lateral border of the tongue.

hybridization stain revealed focal positivity within these cells (Figure 3), leading to a diagnosis of OHL. Laboratory evaluation demonstrated a normal complete blood cell count with differential and liver panel as well as a negative HIV test. The patient otherwise felt well and denied fevers, lymphadenopathy, and weight loss.

We consulted with an infectious disease and immunodeficiency specialist regarding the patient's case. Before conducting further evaluation beyond HIV screening for immunodeficiency states, adalimumab was discontinued to see if the OHL would spontaneously resolve. Three months after discontinuation of adalimumab, the white plaque on the right lateral tongue was notably improved. The OHL continued to disappear and was completely resolved 1 year after discontinuation of adalimumab. The patient's psoriasis had subsequently remained well controlled with diet and weight loss, smoking cessation, topical steroids, and apremilast without any recurrence of the OHL.

Oral hairy leukoplakia is associated with upregulated EBV replication and EBV-encoded proteins such as latent membrane protein $1 .{ }^{2}$ It often presents as white or gray patches on the lateral lingual margins with prominent folds and/or projections, giving a shaggy appearance. Oral hairy leukoplakia often is specific for HIV infection and rarely is associated with other immunodeficiencies. ${ }^{2}$ Prasad and Bilodeau ${ }^{5}$ performed a literature review of medical conditions and immunosuppressive medications associated with OHL in patients without HIV. Allogeneic transplant was associated with the highest incidence of OHL in HIVnegative patients $\left(59.2 \%\right.$ [45/76]). ${ }^{5}$ Various combinations of immunosuppressive medications (eg, prednisone, cyclosporine, azathioprine) also may be implicated in cases of HIV-negative patients with OHL. A case of OHL also has been reported with long-standing use of inhaled corticosteroids in an immunocompetent, HIV-negative patient. ${ }^{6}$ Another case was reported with long-term use of the aromatic antiepileptic lamotrigine, which resolved once
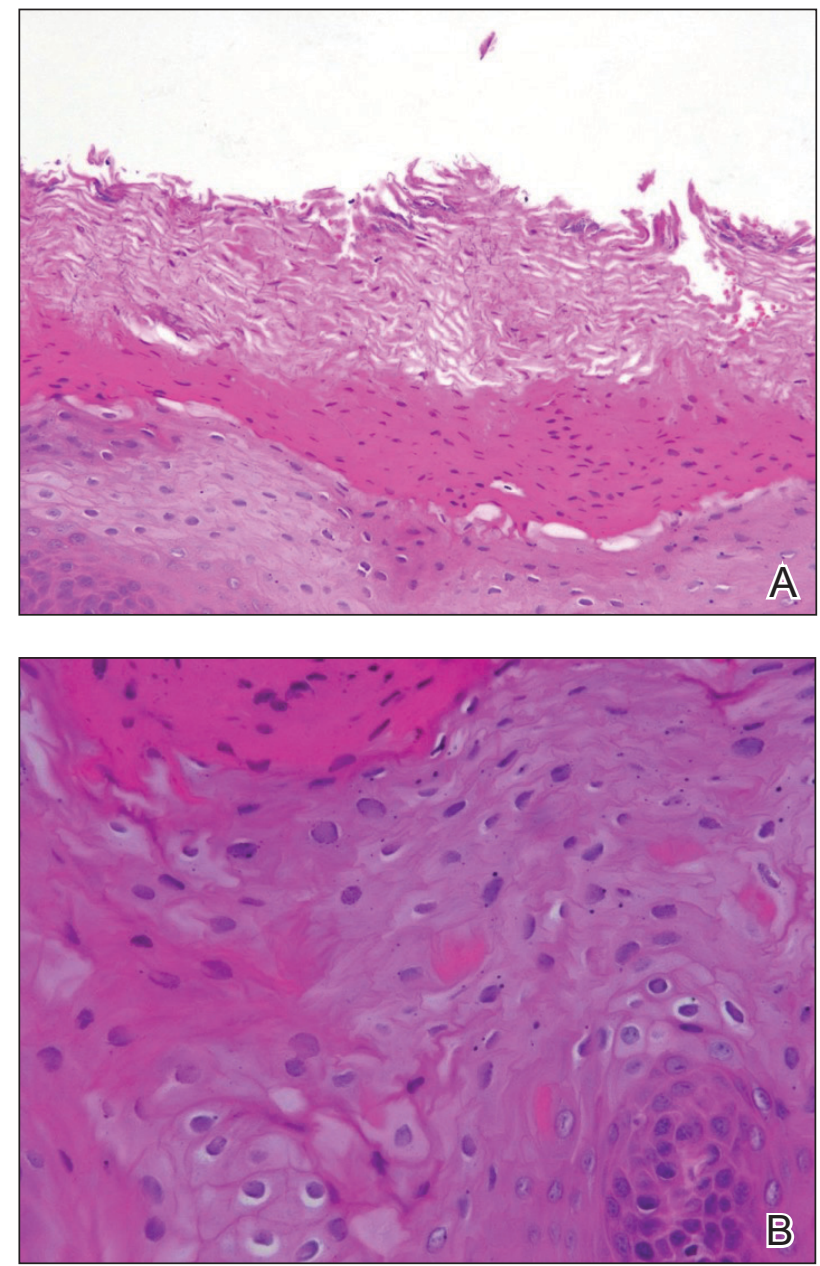

FIGURE 2. A, Histopathology revealed hyperkeratosis containing Candida organisms (H\&E, original magnification $\times 200$ ). B, Highpower view exhibited cells with nuclear chromatin margination and inclusions in the superficial spinous layer (H\&E, original magnification $\times 400)$.

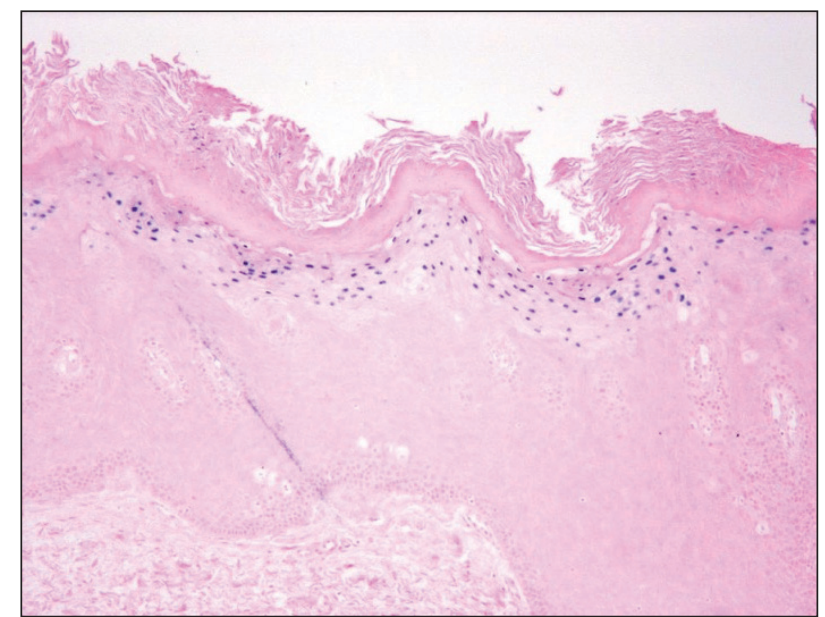

FIGURE 3. Epstein-Barr virus (EBV) DNA in situ hybridization demonstrated blue nuclei staining positively for EBV within the superficial spinous layer (original magnification $\times 100$ ). 
stopping the medication. ${ }^{8}$ Although EBV is an oncovirus and has been associated with lymphoproliferative disorders and nasopharyngeal carcinoma, OHL is not considered to be a premalignant lesion. ${ }^{7}$ Despite the strong association between OHL and HIV, our patient was HIV negative. The only immunocompromising factor in our patient was the use of adalimumab to treat psoriasis. We did not conduct further testing for immunodeficiency states because the OHL spontaneously resolved when the adalimumab was discontinued.

PubMed and Ovid searches of articles indexed for MEDLINE using the terms adalimumab and oral hairy leukoplakia as well as TNF-alpha inhibitor and oral hairy leukoplakia with humans and English language as limitations revealed that no cases have been reported in the literature demonstrating an association between OHL and adalimumab or any other TNF- $\alpha$ inhibitor. However, Cetkovska et al ${ }^{9}$ reported a case of EBV hepatitis and subsequently chronic hepatitis as a complication of infliximab used for the treatment of chronic psoriasis. Because TNF- $\alpha$ and IFN- $\gamma$ play an important role in controlling viral infections, there is an increased risk for reactivating a viral illness when depleting TNF through pharmacologic measures (ie, adalimumab, infliximab). ${ }^{8}$ Another case of EBV-associated plasmablastic lymphoma was reported after 1 year of adalimumab use in a patient with Crohn disease. The plasmablastic lymphoma resolved after 4 rounds of chemotherapy. ${ }^{10}$

The only contraindication for the use of adalimumab is a known hypersensitivity to the drug. Relative contraindications for use of adalimumab include active tuberculosis, demyelinating disease, hematologic diseases (ie, thrombocytopenia, pancytopenia), lymphoma, hepatitis C, and hepatitis B. ${ }^{11}$ The most common adverse effect of adalimumab is an injection-site reaction. Additional reported adverse effects of TNF- $\alpha$ inhibitors as a class are lymphoma, melanoma, nonmelanoma skin cancer, reactivation of latent tuberculosis, congestive heart failure, autoimmunity, and hematologic toxicity. ${ }^{11}$

This case demonstrates an association between adalimumab and OHL in an HIV-negative patient. Although the mechanism behind OHL and immunosuppression remains to be elucidated, this association is important to keep in mind when using adalimumab or other TNF- $\alpha$ inhibitors for the treatment of psoriasis or other medical conditions.

\section{REFERENCES}

1. Triantos D, Porter SR, Scully C, et al. Oral hairy leukoplakia: clinicopathologic features, pathogenesis, diagnosis, and clinical significance. Clin Infect Dis. 1997;25:1392-1396.

2. Greenspan D, Greenspan JS, Conant M, et al. Oral "hairy" leucoplakia in male homosexuals: evidence of association with both papillomavirus and a herpes-group virus. Lancet, 1984;2:831-834.

3. Glick M, Muzyka BC, Lurie D, et al. Oral manifestations associated with HIV-related disease as marks for immune suppression and AIDS. Oral Surg Oral Med Oral Pathol. 1994;77:344-349.

4. Chambers AE, Conn B, Pemberton M, et al. Twenty-first-century oral hair leukoplakia - a non-HIV-associated entity. Oral Surg Oral Med Oral Patho Oral Radiol. 2015;119:326-332.

5. Prasad JL, Bilodeau EA. Oral hairy leukoplakia in patients without HIV: presentation of 2 new cases. Oral Surg Oral Med Oral Pathol Oral Radiol. 2014;118:E151-E160.

6. Moffat M, Jauhar S, Jones ME, et al. Oral hairy leukoplakia in an HIVnegative, immunocompetent patient. Oral Biosci Med. 2005;2:282-284.

7. Greenspan JS, Greenspan D. Oral hairy leukoplakia: diagnosis and management. Oral Surg Oral Med Oral Pathol. 1989;67:396-403.

8. Gordins P, Sloan P, Spickett GP, et al. Oral hairy leukoplakia in a patient on long-term anticonvulsant treatment with lamotrigine. Oral Surg Oral Med Oral Pathol Oral Radiol Endod. 2011;111:E17-E23.

9. Cetkovska P, Lomicova I, Mukensnabl P, et al. Anti-tumour necrosis factor treatment of severe psoriasis complicated by Epstein-Barr virus hepatitis and subsequently by chronic hepatitis. Dermatol Ther. 2015;28:369-372

10. Liu L, Charabaty A, Ozdemirli M. EBV-associated plasmablastic lymphoma in a patient with Crohn's disease after adalimumab treatment. J Crohns Colitis. 2013;7:E118-E119.

11. Humira [package insert]. North Chicago, IL: AbbVie Inc; 2018. 